\title{
Squeezing bandwidth controllable twin beam light and phase sensitive nonlinear interferometer based on atomic ensembles
}

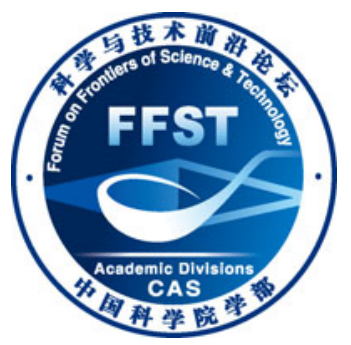

\author{
JING JieTai $^{1,2}$, LIU CunJin ${ }^{1,2}$, ZHOU ZhiFan ${ }^{1,2}$, HUDELIST Florian ${ }^{1,2}$, YUAN ChunHua ${ }^{1}$, \\ CHEN LiQing ${ }^{1,2}$, LI XiaoYun ${ }^{1}$, QIAN Jing ${ }^{1}$, ZHANG KeYe ${ }^{1}$, ZHOU Lu ${ }^{1}$, MA HongMei ${ }^{1}$, \\ DONG GuangJiong ${ }^{1,2}$, OU ZeYu ${ }^{1,2,3}$ \& ZHANG WeiPing ${ }^{1,2^{*}}$
}

\footnotetext{
${ }^{1}$ Quantum Institute for Light and Atoms, Department of Physics, East China Normal University, Shanghai 200062, China;

${ }^{2}$ State Key Laboratory of Precision Spectroscopy, East China Normal University, Shanghai 200062, China;

${ }^{3}$ Department of Physics, Indiana University-Purdue University Indianapolis, Indianapolis, Indiana 46202, USA
}

Received December 12, 2011; accepted February 13, 2012

\begin{abstract}
We review our recent experimental progress in quantum technology employing amplification effect of four-wave mixing in a rubidium vapor. We have produced an intensity difference squeezed light source at frequencies as low as $1.5 \mathrm{kHz}$ which is so far the lowest frequency at which squeezing has been observed in an atomic system. Moreover, we find that the bandwidth of our squeezed light source can be controlled with light intensity pumping. Using our non-classical light source, we have further developed a nonlinear Mach-Zehnder (MZ) interferometer, for which the maximum fringe intensity depends quadratically on the intensity of the phase-sensing field at the high-gain regime, leading to much better sensitivity than a linear MZ interferometer in which the beam splitters have the same phase-sensing intensity. The quantum technologies developed by our group could have great potential in areas such as precision measurement and quantum information.
\end{abstract}

atomic ensemble, four wave mixing, quantum light source, nonlinear interferometer

Citation: Jing J T, Liu C J, Zhou Z F, et al. Squeezing bandwidth controllable twin beam light and phase sensitive nonlinear interferometer based on atomic ensembles. Chin Sci Bull, 2012, 57: 1925-1930, doi: 10.1007/s11434-012-5101-7

Photons are natural fast carriers of quantum information. For this reason, much attention [1,2], particularly over the last two decades, has been drawn towards applications of quantum optical systems to quantum information processing, such as quantum communication and distributed quantum networks. The research necessitates high-quality quantum light sources that generate squeezed light with large noise suppression below the shot noise level (SNL) and entangled states.

In 1985, Slusher et al. pioneered squeezed light generation based on four-wave mixing (FWM) in an optical cavity [3]. Since then, several methods have been explored to obtain squeezed states; a fundamental and frequently-used

\footnotetext{
*Corresponding author (email: wpzhang@phy.ecnu.edu.cn)
}

technique is the optical parametric oscillator consisting of nonlinear crystals and cavities to build up non-classical states very efficiently [4-7]. Using such methods, very strong single mode squeezing has been realized in the past few years. For example, vacuum squeezing levels of $-10 \mathrm{~dB}$ were achieved in 2008 [8] and Mehmet et al. obtained up to $-11.5 \mathrm{~dB}$ squeezing last year [9]. By contrast, without any optical cavity and mode cleaner, FWM has gradually become another popular method because of its simple experimental setup. Since the initial work of Lett's group in 2007 [10], as much as $-9.2 \mathrm{~dB}$ squeezing has been reported in hot rubidium vapor [11]. Based on FWM, a pair of multi-spatialmode beams was produced, carrying two images which are in non-separable continuous-variable entangled states. The images were composed of "squeezed vacuum" twin beams 
that are strongly entangled when projected onto a range of different spatial local oscillator modes [12].

Squeezing bandwidth and low frequency squeezing are important characteristics of squeezed photonic states for their potential applications. Squeezed light with low frequency was first proposed to be used in high-sensitivity detection [13]. Up to now, many groups have successfully generated low frequency squeezing at the sub-megahertz range [14-17]. Recently, low-frequency squeezing was found to be interesting for electromagnetically-induced transparency-based quantum information protocols and other applications at atomic transition wavelengths [16]. Our work [17] is derived from this motivation and inspired by previous work such as the creation of beams with a low-frequency quantum correlation based on FWM in a hot rubidium vapor [18]. We have generated an intensity difference squeezed light source at frequencies as low as $1.5 \mathrm{kHz}$ which is so far the lowest frequency at which squeezing has been observed in an atomic system. Furthermore, we demonstrate that we can use pumping light intensity to control the bandwidth of our squeezed light source.

The quantum light source generated in our experiment could have great potential in the application of precise metrology and quantum information. Therefore, we have extended our quantum light source to realize a nonlinear MZ interferometer [19], which was proposed by Yurke et al. in 1986 [20]. In contrast with previous optical interferometers using linear optical processing, our experiment shows that the maximum fringe intensity of the nonlinear $\mathrm{MZ}$ interferometer has a quadratic relation to the intensity of the phase sensing field in the high gain regime, and thus has great potential to reach the ultimate quantum limit of phase measurement, i.e. the Heisenberg limit [21].

In the following, we present a review on our experimental progress of a high quality quantum light source [17] and a nonlinear MZ interferometer [19]. This paper is organized as follows. Section 1 introduces our recent progress in quantum light source using the FWM process in rubidium vapor atomic ensemble. The nonlinear interferometer using the FWM process as beam splitters is reviewed in Section 2. Conclusions are summarized in Section 3.

\section{Quantum light source}

In this section, we report the realization of low-frequency and controllable-bandwidth squeezing based on a nondegenerate FWM process in a hot rubidium vapor at the optical D1 transition.

We performed our experiment using a Ti:sapphire laser (Spectra-Physics) tuned about $1 \mathrm{GHz}$ to the blue of the D1 line of rubidium $\left(5 \mathrm{~S}_{1 / 2} \rightarrow 5 \mathrm{P}_{1 / 2}, 795 \mathrm{~nm}\right)$ with a line width of about $30 \mathrm{kHz}$. This laser supplies coherent light used to interact with rubidium atoms in a hot vapor cell, resulting in a strong FWM, or nonlinear phase insensitive amplification process and generates correlated twin beams, the probe and the conjugate, with a $-6 \mathrm{GHz}$ frequency difference.

Figure 1 shows the energy diagram of the ${ }^{85} \mathrm{Rb} \mathrm{D} 1$ line which forms a double- $\lambda$ system and the schematic diagram of the experimental setup. The output power of the laser is $1 \mathrm{~W}$. A polarizing beam splitter (PBS) is used to split the beam into a weak seed probe beam and a much stronger pump beam. The seed beam is red, detuned about $3 \mathrm{GHz}$ using an acousto-optic modulator (AOM) (Brimrose). The AOM is driven by an RF signal generator (Agilent, N9310A). The polarizations of the pump and probe are chosen perpendicular to each other, so the pump field can be filtered out after the vapor cell with a Glan-Thompson polarizer, which is used to combine the weak probe and strong pump with an angle of $0.4^{\circ}$. The crossing point is in the center of a $12 \mathrm{~mm}$ long vapor cell which is filled with isotopically-pure ${ }^{85} \mathrm{Rb}$ and heated to $120^{\circ} \mathrm{C}$. Both faces of the cell are antireflection coated to achieve a transmission efficiency larger than $98 \%$. The pump beam waist at the crossing point is $550 \mu \mathrm{m}$, whereas the probe beam waist is $300 \mu \mathrm{m}$. The amplified probe after the vapor cell along with the generated conjugate with the same polarization are separated from the pump beam by another Glan-Thompson polarizer with an extinction ratio of $10^{5}: 1$. The probe and conjugate are directly sent to a balanced photodetector (BPD, Thorlabs PDB150) with two high-quantum-efficiency $(96 \%)$ photodiodes. The BPD subtracts the photocurrents with a switchable gain (usually $10^{5} \mathrm{~V} / \mathrm{A}$ ) and sends the signal to a spectrum analyzer to perform a noise level analysis over a certain frequency range.

With a $400 \mathrm{~mW}$ pump and a $10 \mu \mathrm{W}$ probe seed, the
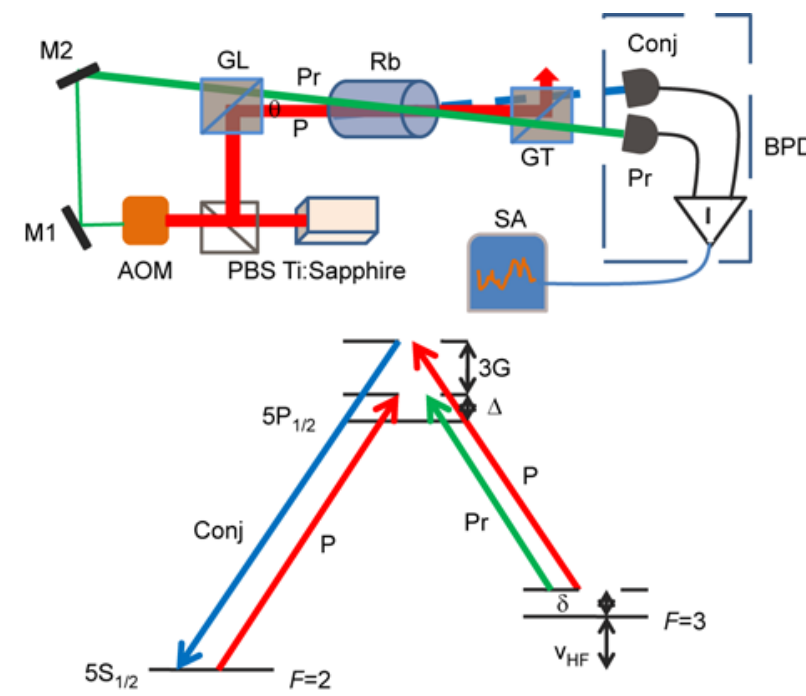

Figure 1 (Color online) Top: experimental setup. PBS, polarizing beam splitter; AOM, acoustic optical modulator; GL, Glan-laser polarizer; GT, Glan-Thompson polarizer; M, mirror; BPD, balanced photodetector; SA, spectrum analyzer. Bottom: energy level diagram of the ${ }^{85} \mathrm{Rb}$ D1 line. P, pump; Pr, probe; Conj, conjugate; $\Delta$, one photon detuning; $\delta$, two photon detuning; $v_{\mathrm{HF}}$, hyperfine structure. 
amplified probe is about $80 \mu \mathrm{W}$ and the conjugate is about $70 \mu \mathrm{W}$. The transmission $(90 \%)$ of the probe is measured by blocking the pump. Both the probe and conjugate are sent to the BPD, the intensity difference noise between the probe and the conjugate is subtracted and then analyzed by a spectrum analyzer (Agilent E4411B), as seen in Figure 2. To measure the SNL, we guide a coherent laser beam of power $150 \mu \mathrm{W}$, equivalent to the total power of the probe and conjugate, splitting it 50/50 and sending the resulting beams to the BPD. The measurement is taken with $100 \mathrm{kHz}$ resolution bandwidth (RBW) and $1 \mathrm{kHz}$ video bandwidth (VBW).

We investigated the squeezing properties in the lowfrequency region with the pump power set to $400 \mathrm{~mW}$. We use a fast Fourier transform (FFT) spectrum analyzer (SRS SR770) with bandwidth spanning from the DC to $100 \mathrm{kHz}$. As seen in Figure 3, we observe the relative intensity squeezing of an $80 \mu \mathrm{W}$ probe and a $70 \mu \mathrm{W}$ conjugate at frequencies as low as $1.5 \mathrm{kHz}$. This is, to the best of our knowledge, $1 \mathrm{kHz}$ lower than the previous best result obtained from an atomic system at this wavelength. The RBW of the FFT spectrum analyzer is $31.25 \mathrm{~Hz}$ for this measurement.

Another important characteristic is the squeezing bandwidth. A large bandwidth is always useful for communications; in particular, squeezed light has been proven to be a

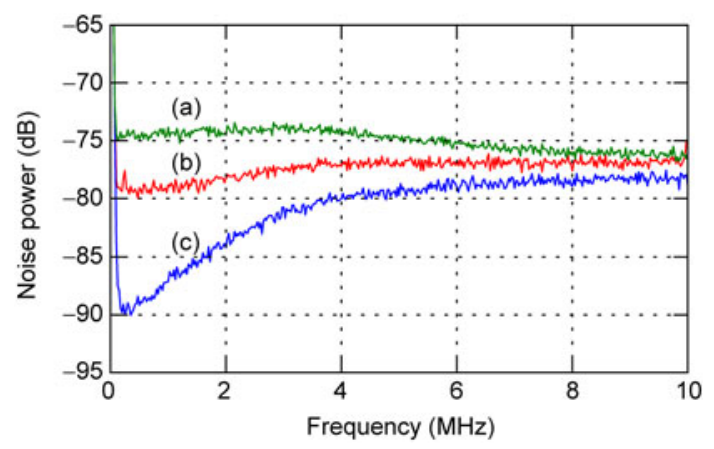

Figure 2 (Color online) Noise power. (a) SNL, (b) intensity difference squeezing between the probe and the conjugate, and (c) the background noise level. $100 \mathrm{kHz}$ RBW and $1 \mathrm{kHz}$ VBW.

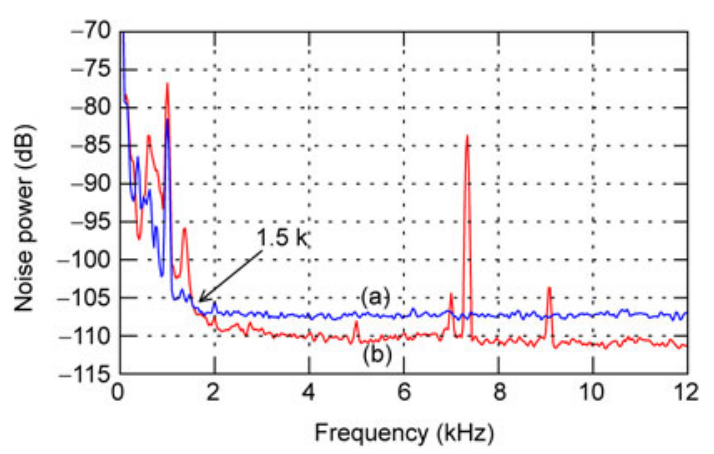

Figure 3 (Color online) Low-frequency noise power spectrum from the FFT spectrum analyzer with $31.25 \mathrm{~Hz} \mathrm{RBW}$. (a) SNL and (b) intensity difference squeezing between the probe and the conjugate. good source for quantum communication. Most likely, squeezed states with large bandwidth will be good candidates for future high efficient quantum communication. In our experiment system, we found the pump power has a capacity to control the squeezing bandwidth of this FWM amplifier. We change the pump power from 100 to $700 \mathrm{~mW}$ with the other parameters fixed and measure the squeezing bandwidth as shown in Figure 4 We can see a strong linear relationship between the squeezing bandwidth and the pump power.

\section{Nonlinear Mach-Zehnder interferometer}

A simple interferometer such as the Mach-Zehnder interferometer shown in Figure 5(a) consists of two beam splitters with one acting as a wave splitter and the other as wave combiner. For simplicity of argument, we take these as being identical with the same transmissivity $\mathrm{T}$ and reflectivity $\mathrm{R}$. From any standard optics textbook, the output intensity is related to the input by

$$
I_{\text {out }}=2 I_{0} \operatorname{TR}(1+\cos \varphi) / 2=I_{\mathrm{F}}(1+\cos \varphi) / 2,
$$

where $I_{0}$ is the input intensity and $I_{\mathrm{F}}=4 I_{0} T R$ is the maximum fringe intensity. The best sensitivity for measuring a small phase change occurs at $\varphi=\pi / 2$ with

$$
\Delta I_{\text {out }}=I_{\mathrm{F}} \frac{\Delta \varphi}{2}=2 T I_{\mathrm{ps}} \Delta \varphi,
$$

where $I_{\mathrm{ps}}=R I_{0}$ is the intensity of the field subject to a phase change (phase-sensing field). Thus, the sensitivity for phase measurement is directly related to the fringe intensity $I_{\mathrm{F}}$ and for a linear interferometer is also proportional to the phase sensing intensity.

Moreover, there are some nonlinear processes that are phase sensitive. Yurke et al. proposed in 1986 [20] to use parametric processes to measure phases and ultimate quantum
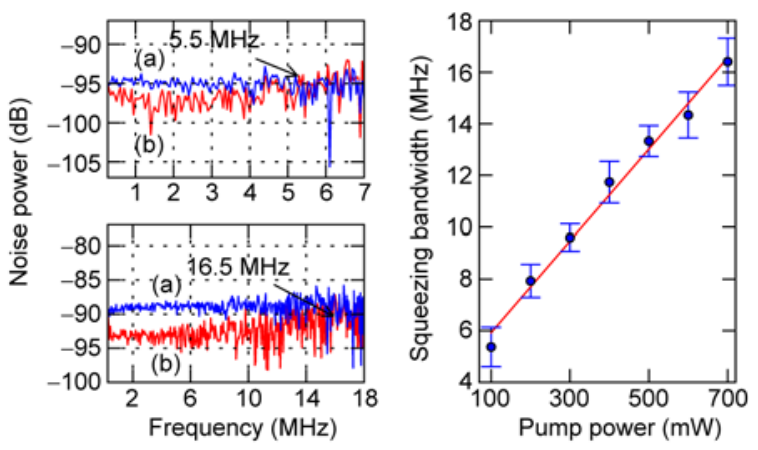

Figure 4 (Color online) Left: squeezing with pump power at $100 \mathrm{~mW}$ (top) and $700 \mathrm{~mW}$ (bottom). (a) shows SNL and (b) is squeezing. Right: squeezing bandwidth versus pump power with measured data (dots) in our experiment and linear fit of the data (straight line). $100 \mathrm{kHz} \mathrm{RBW}$ and $1 \mathrm{kHz}$ VBW. 
limits, i.e. the Heisenberg limit, can be achieved in sensitivities of phase measurements [21]. There were numerous theoretical analyses on such a system, including the recent Plick et al. analysis suggesting a further boost from a coherent state injection [22]. However, since its inception, there has been no experimental realization perhaps because the output field in the original proposal by Yurke et al. is very weak to detect at experimentally-controllable gain. In this part, we report on an experiment in which we construct a nonlinear interferometer with parametric amplifiers acting as beam splitters to split and recombine an incoming optical field. If properly balanced, the interferometer can in principle have $100 \%$ visibility. Since amplification is actively involved in the interferometer, the phase sensing field inside the interferometer is amplified from the input field and so is the output field exhibiting the interference fringe. Thus, the sensitivity can be greatly enhanced as compared to the traditional linear interferometer.

Consider the schematic sketch in Figure 5(b). Our interferometer consists of two parametric amplifiers, PA1 and PA2. The first amplifies the input "signal" field and generates a conjugate "idler" field; thus, PA1 serves as a beam splitter. The second serves as a beam combiner that mixes amplified signal and fields. The action of a parametric amplifier is well described in any nonlinear optics text book [23] as

$$
A_{\mathrm{s}}^{\text {out }}=G A_{\mathrm{s}}^{\text {in }}+g A_{\mathrm{i}}^{\text {in* }} ; A_{\mathrm{i}}^{\text {out }}=G A_{\mathrm{i}}^{\text {in }}+g A_{\mathrm{s}}^{\text {in }},
$$

where s and i stand for signal and idler, respectively; $G$ is the amplitude gain of the amplifier; and $|G|^{2}-|g|^{2}=1$. Therefore, if there is no idler field input at PA1, the fields after PA1 is

$$
A_{\mathrm{s} 1}=G_{1} A_{\mathrm{s} 0} ; A_{\mathrm{i} 1}=g_{1} A_{\mathrm{s} 0}^{*} .
$$

Here, $A_{\mathrm{s} 0}$ is the input signal field. Note that when the gain is large, $G_{1} \approx g_{1}>>1$, and we have a nearly-equal splitting of $A_{\mathrm{s} 0}$ but the split fields are amplified from the input. Assuming the idler field is subject to a phase shift of $\varphi$, we obtain from eqs. (3) and (4) the PA2 outputs

(a)

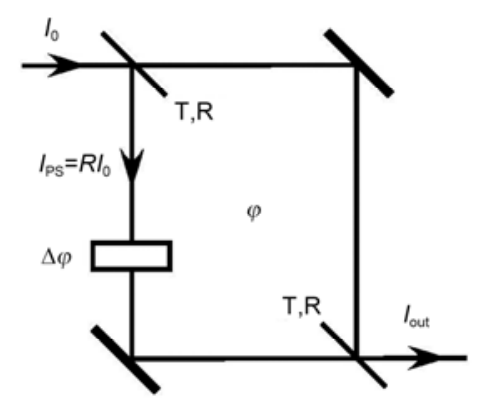

(b)

$$
A_{\mathrm{s} 2}=G(\varphi) A_{\mathrm{s} 0} ; A_{\mathrm{i} 2}=g(\varphi) A_{\mathrm{s} 0}^{*}
$$

with

$$
G(\varphi)=G_{1} G_{2}+g_{1}^{*} g_{2} \mathrm{e}^{-i \phi} ; g(\varphi)=G_{1}^{*} g_{2}+G_{2} g_{1} \mathrm{e}^{i \phi} .
$$

If both amplifiers have the same gain: $G_{1}=G_{2}=G_{0} ; g_{1}=g_{2}=g_{0}$, we have $\left(G_{0}, g_{0}=\right.$ real $)$

$$
G(\varphi)=1+\left|g_{0}\right|^{2}\left(1+\mathrm{e}^{-i \phi}\right) ; g(\varphi)=G_{0} g_{0}\left(1+\mathrm{e}^{i \phi}\right) .
$$

From eq. (4), we find the intensity of the phase sensing field (idler 1) inside the interferometer as

$$
I_{\mathrm{i} 1}=\left|A_{\mathrm{i} 1}\right|^{2}=\left|g_{0}\right|^{2} I_{0}
$$

with $I_{0}=\left|A_{\mathrm{s} 0}^{2}\right|$ as the input intensity. The intensities of the output fields can also be easily obtained as

$$
I_{\mathrm{s} 2}=I_{0}\left[1+2\left|G_{0} g_{0}\right|^{2}(1+\cos \varphi)\right], I_{\mathrm{i} 2}=2 I_{0}\left|G_{0} g_{0}\right|^{2}(1+\cos \varphi) \text {. }
$$

Thus both the output fields show interference fringes when the phase is scanned. The idler side always has a visibility of $100 \%$, but the signal side's visibility is

$$
V_{\mathrm{s}}=2\left|G_{0} g_{0}\right|^{2} /\left(1+2\left|G_{0} g_{0}\right|^{2}\right) \text {, }
$$

which is close to $100 \%$ if $G_{0} \approx g_{0}>>1$. From eq. (9), we find the maximum fringe intensity at the idler output to be

$$
I_{\mathrm{i} 2}^{\mathrm{M}}=4 I_{0}\left|G_{0} g_{0}\right|^{2}=4\left|G_{0}\right|^{2} I_{\mathrm{ps}}=4\left(I_{\mathrm{ps}}+I_{0}\right) I_{\mathrm{ps}} / I_{0} .
$$

Here $I_{\mathrm{ps}} \equiv I_{\mathrm{i} 1}$ is the intensity of the phase sensing field. For a large gain and a fixed input $I_{0}$, we have $I_{\mathrm{ps}}>>I_{0}$ and $I_{\mathrm{i} 2}^{\mathrm{M}} \infty I_{\mathrm{ps}}^{2}$. Eq. (11) is the main feature of this nonlinear interferometer that is different from a traditional linear interferometer, i.e., the maximum fringe intensity depends quadratically on the phase-sensing intensity, in contrast to the linear dependence in eq. (1) for a linear interferometer. Furthermore, the maximum fringe intensity is amplified from the phase sensing intensity by a factor of $4\left|G_{0}\right|^{2}$, giving an enhancement in sensitivity as compared to eq. (2) for a linear interferometer.

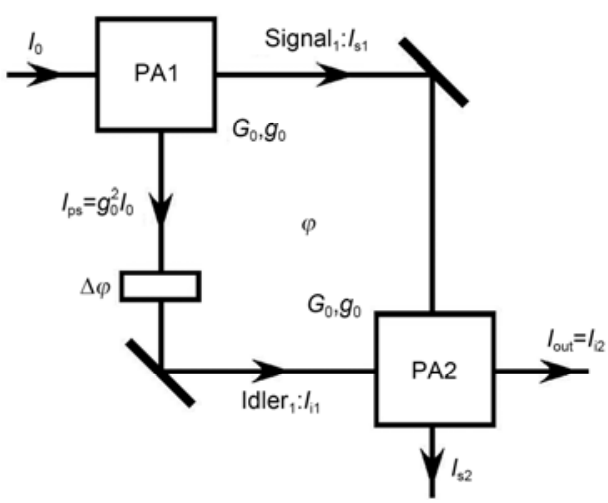

Figure 5 (a) A linear Mach-Zehnder interferometer. (b) A nonlinear interferometer with parametric amplifiers (PA1 and PA2) as the equivalent beam splitters. 
The experimental layout is shown in Figure 6. We use two identical $\mathrm{Rb}$ vapor cells as two parametric amplifiers based on the FWM process. Initially we have all the experimental parameters mentioned in Section 1 to obtain a high-intensity difference squeezing of signal and idler, which means producing highly-correlated twin beams. Next, using a $4 \mathrm{f}$ imaging system, we couple the outputs of the first vapor cell (the amplified signal and the conjugate idler beams) into the second vapor cell through another Glanlaser polarizer. These are symmetrically crossed with the pump at the center of the second vapor cell at $0.7^{\circ}$, similar to the first vapor cell. The two output beams from the second vapor cell are sent to two photodiodes (D1 and D2) with single-mode fiber coupling for spatial mode clean up. The resulting photocurrents are fed to a digital scope for data collection and analysis. A mirror mounted on a piezoelectric transducer is used to change the phase of the signal and idler beams after the first cell for the phase scan of the interferometer. The relative power of the pump beams can be controlled by half wave plates $\mathrm{H} 1, \mathrm{H} 2$, and $\mathrm{H} 3$ while the power of the injected signal beam by $\mathrm{H} 4$.

Figure 7 shows typical interference fringes at the two outputs of the interferometer. The signal output has a slightly smaller visibility $(94.5 \%)$ than the idler side (98.6\%), as expected from eq. (10). Next, we measure the visibilities at different gains of the parametric amplifier by varying the power of the pump beams. Figure 8 shows the results of the measurement. The red solid line is a plot of eq. (10) multiplied by a factor of 0.95 to account for imperfect alignment of the interferometer. The visibility data of the signal fringe follow this line very well. Moreover, the visibility of the idler fringe is kept constant at around $95 \%$, consistent with eq. (9). To confirm the nonlinear nature of the interferometer, we measure the maximum of the interference fringe of the idler output as a function of the intensity of the phase sensing field (the idler beam by D3 right after the first vapor cell) as we change the pump powers. The results are plotted in Figure 9 in log-log scale. The solid red line is a best fit curve to eq. (11) with $I_{0}$ as the adjustable parameter. The dashed black line is a straight line with a slope of two, showing the quadratic dependence. The inset is in linear scale clearly showing the nonlinear trend. Note that, at the highest value, the maximum fringe intensity is about 30 times the phase sensing intensity.

\section{Conclusions}

With parametric amplification based on FWM in rubidium vapor, we have for the first time demonstrated generation of

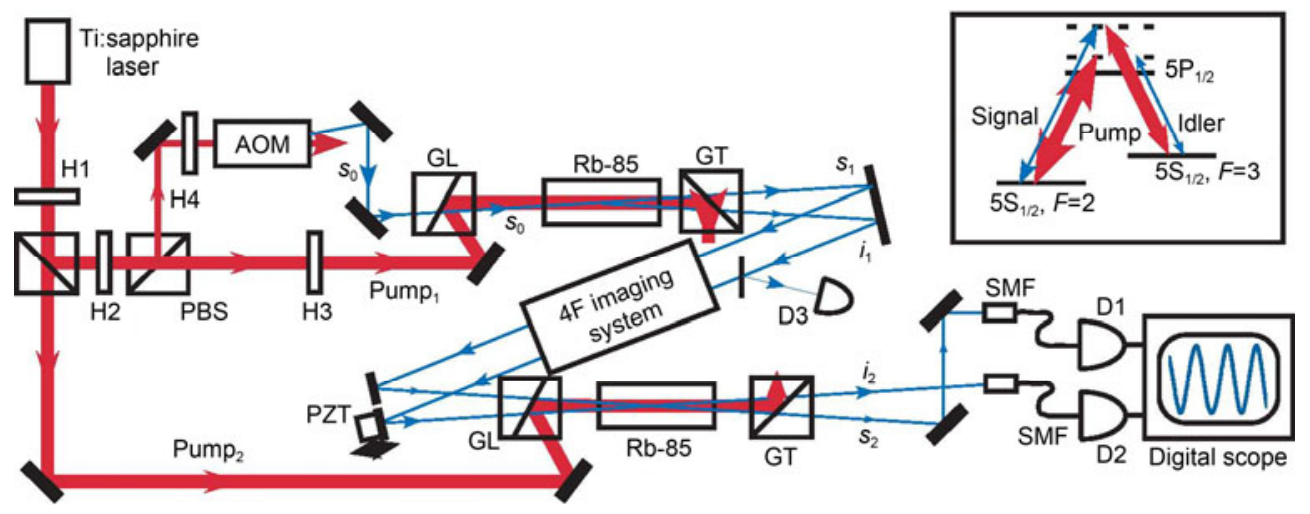

Figure 6 (Color online) Experimental layout of the nonlinear interferometer. H, half wave plate; PBS, polarization beam splitter; AOM, acoustic optic modulator; GL, Glen-laser polarizer; GT, Glen-Thompson polarizer; SMF, single-mode fiber; D, detector.
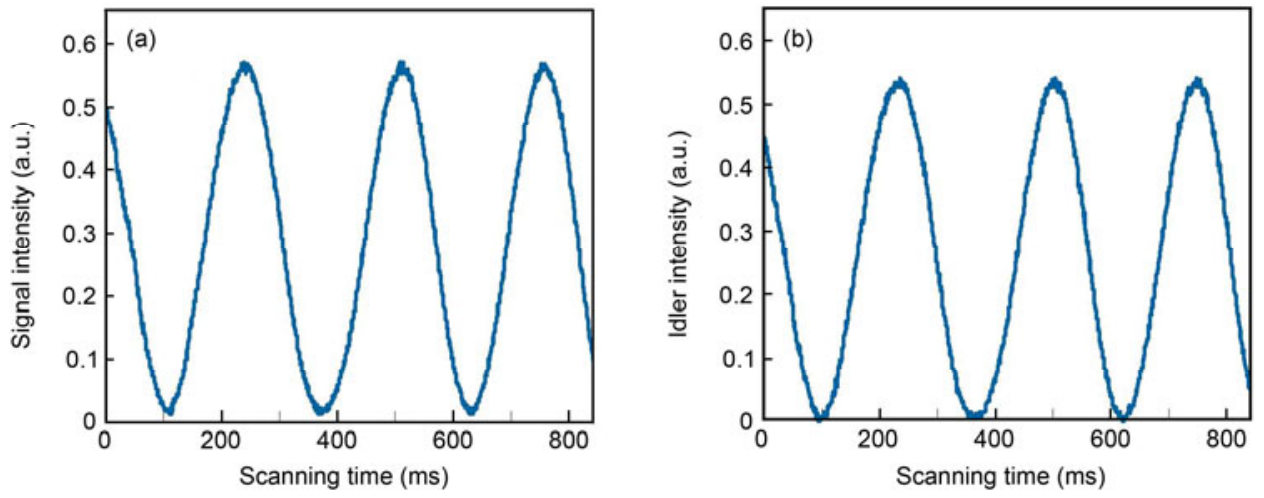

Figure 7 (Color online) Interference fringes for the nonlinear interferometer. (a) Signal output. (b) idler output. 


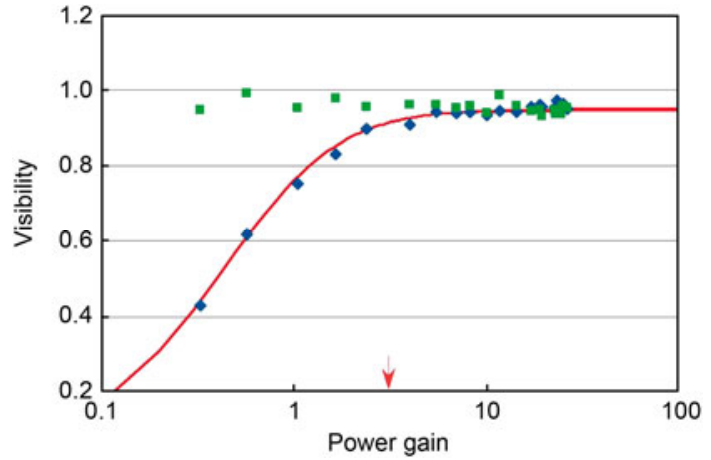

Figure 8 (Color online) Visibility of interference fringe as a function of power gain $\left|g_{0}\right|^{2}$. Green squares, idler fringe; blue diamond, signal fringe; red solid line, plot of $V_{\mathrm{s}}$ in eq. (10). To account for imperfect alignment, $V_{\mathrm{s}}$ is multiplied by a factor of 0.95 . The arrow corresponds to the gain for Figure 3.

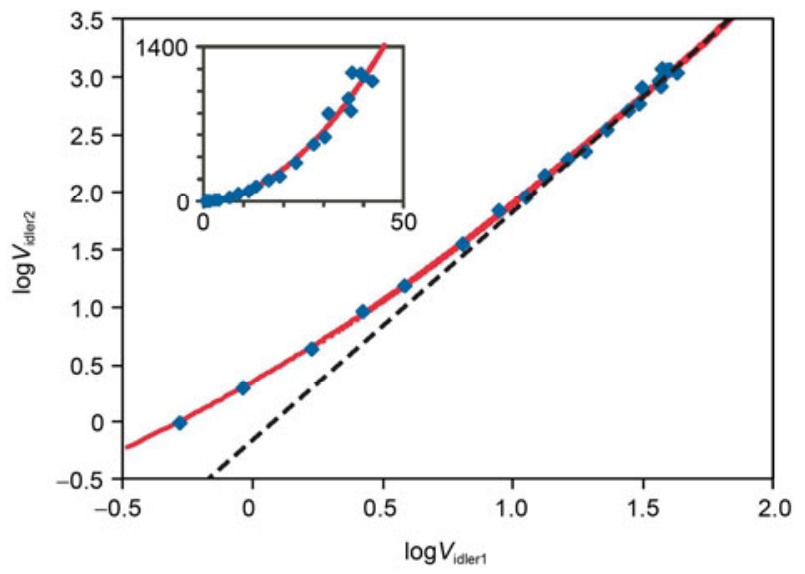

Figure 9 (Color online) Dependence of the maximum intensity of the interference fringe on the intensity of the phase sensing field inside the nonlinear interferometer in a log-log scale plot. Dashed line, slope 2 for quadratic dependence. Inset, linear scale plot; red solid line, fit to the function in eq. (11).

strong correlated twin beams with $-5 \mathrm{~dB}$ intensity difference squeezing. With squeezing frequencies was as low as $1.5 \mathrm{kHz}$, the squeezing bandwidth was completely controllable. Further, using two cascade FWM processes, we constructed a nonlinear $\mathrm{MZ}$ interferometer that has much higher phase sensitivity than a linear MZ interferometer.

Our experimental achievement of a high quality quantum light source and a nonlinear MZ interferometer could be of potential application in ultra-high precision measurements and quantum information processing, as well as ultracold atom experiments.

This work was supported by the National Basic Research Program of China (2011CB921604 and 2011CB921602), the National Natural Science Foundation of China (10974057, 11004057, 11004058, 11004059, 11034002 and 10874045), Shanghai Pujiang Program (09PJ1404400), the Program for Professor of Special Appointment (Eastern Scholar) at Shanghai Institutions of Higher Learning, the Program for New Century Excellent Talents in University (NCET-10-0383), "Shu Guang” Project of Shanghai Municipal Education Commission and Shanghai Education Development Foundation (11SG26), the Scientific Research Foundation for the Returned Overseas Chinese Scholars (State Education Ministry), the "Chen Guang" Project of Shanghai Municipal Education Commission and the Shanghai Education Development Foundation (10CG24).

1 Kok P, Munro W J, Nemoto K, et al. Linear optical quantum computing with photonic qubits. Rev Mod Phys, 2007, 79: 135-174

2 Sangouard N, Simon C, de Riedmatten H, et al. Quantum repeaters based on atomic ensembles and linear optics. Rev Mod Phys, 2011, 83: 33-80

3 Slusher R E, Hollberg L W, Yurke B, et al. Observation of squeezed states generated by four-wave mixing in an optical cavity. Phys Rev Lett, 1985, 55: 2409-2412

4 Wu L, Kimble H J, Hall J L, et al. Generation of squeezed states by parametric down conversion. Phys Rev Lett, 1986, 57: 2520-2523

5 Li X Y, Pan Q, Jing J T, et al. Quantumdense coding exploiting a bright Einstein-Podolsky-Rosen beam. Phys Rev Lett, 2002, 88: 047904

6 Jing J T, Zhang J, Yan J, et al. Experimental demonstration of tripartite entanglementand controlled dense coding for continuous variables. Phys Rev Lett, 2003, 90: 167903

7 Jia X J, Su X, Pan Q, et al. Experimental demonstration of unconditional entanglement swapping for continuous variables. Phys Rev Lett, 2004, 93: 250503

8 Vahlbruch H, Mehmet M, Chelkowski S, et al. Observation of squeezed light with $10 \mathrm{~dB}$ quantum-noise reduction. Phys Rev Lett, 2008, 100: 033602

9 Mehmet M, Vahlbruch H, Lastzka N, et al. Observation of squeezed states with strong photon-number oscillations. Phys Rev A, 2010, 81: 013814

10 McCormick C F, Boyer V, Arimondo E, et al. Strong relative intensity squeezing by four-wave mixing in rubidium vapor. Opt Lett, 2007, 32: 178-180

11 Glorieux Q, Guidoni L, Guibal S, et al. Strong quantum correlations in four wave mixing in ${ }^{85} \mathrm{Rb}$ vapor. Proc SPIE, 2010, 7727: 772703

12 Boyer V, Marino A M, Pooser R C, et al. Entangled images from four-wave mixing. Science, 2008, 321: 544-547

13 Caves C M. Quantum-mechanical noise in an interferometer. Phys Rev D, 1981, 23: 1693-1708

14 McKenzie K, Grosse N, Bowen W P, et al. Squeezing in the audio gravitational-wave detection band. Phys Rev Lett, 2004, 93: 161105

15 Chelkowski S, Vahlbruch H, Danzmann K, et al. Coherent control of broadband vacuum squeezing. Phys Rev A, 2007, 75: 043814

16 Mikhailov E E, Novikova I. Low-frequency vacuum squeezing via polarization self-rotation in Rb vapor. Opt Lett, 2008, 33: 1213-1215

17 Liu C J, Jing J T, Zhou Z F, et al. Realization of low frequency and controllable bandwidth squeezing based on a four-wave-mixing amplifier in rubidium vapor. Opt Lett, 2011, 36: 2979-2981

18 McCormick C F, Morino A M, Boyer V, et al. Strong low-frequency quantum correlations from a four-wave-mixing amplifier. Phys Rev A, 2008, 78: 043816

19 Jing J T, Liu C J, Zhou Z F, et al. Realization of a nonlinear interferometer with parametric amplifiers. Appl Phys Lett, 2011, 99: 011110

20 Yurke B, McCall S L, Klauder J R. SU(2) and SU(1,1) interferometers. Phys Rev A, 1986, 33: 4033-4054

21 Ou Z Y. Complementarity and fundamental limit in precision phase measurement. Phys Rev Lett, 1996, 77: 2352-2355

22 Plick W N, Dowling J P, Agarwal G S. Coherent-light-boosted, subshot noise, quantum interferometry. New J Phys, 2010, 12: 083014

23 Boyd R. Nonlinear Optics. New York: Academic Press, 1992

Open Access This article is distributed under the terms of the Creative Commons Attribution License which permits any use, distribution, and reproduction in any medium, provided the original author(s) and source are credited. 